NAGW 1340

$1 \lambda-90-C R$

FERMILAB-Pub-91/186-A

UTAP-128/91

July, 1991

\title{
Phase transitions triggered by quantum fluctuations in the inflationary universe
}

\author{
MichiYasu Nagasawa ${ }^{1}$ AND JUn'IChI YoKOYAMA ${ }^{1,2}$ \\ ${ }^{1}$ Department of Physics, Faculty of Sciences, \\ The University of Tokyo, Tokyo 113, Japan \\ ${ }^{2}$ NASA/Fermilab Astrophysics Center, \\ Fermi National Accelerator Laboratory, Batavia, IL60510, USA
}

ABSTRACT

The dynamics of a second-order phase transition during inflation, which is induced by timevariation of spacetime curvature, is studied as a natural mechanism to produce topological defects of typical grand unification scales such as cosmic strings or global textures. It is shown that their distribution is almost scale-invariant with small- and large-scale cutoffs. Also discussed is how these cutoffs are given.

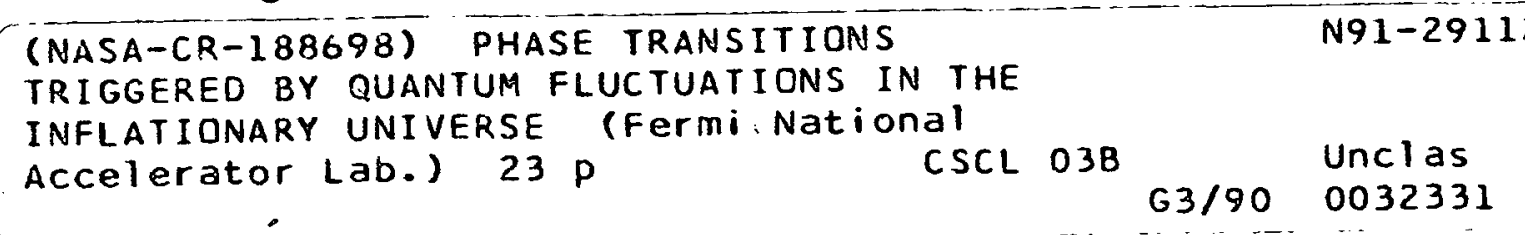




\section{Introduction}

The idea of spontaneous symmetry breaking plays an important role in constructing unified theories of elementary interactions. At ultrahigh temperatures of order of their unification scale, it is believed that the presently broken symmetries are restored due to high-temperature correction to the potential of Higgs fields which determines the symmetry of the system. Unfortunately, however, it is hardly possible to verify their predictions experimentally, since the energy scale is higher than the accessible scales by many orders of magnitude in a laboratory.

Trying to get out of such a frustrating situation, many people applied these theories to the early universe whose temperature, according to the conventional big bang cosmology, was once so high as the unification energy scale. One of their natural consequences is that the universe has presumably undergone a number of thermal phase transitions in the course of its early evolution, in some of which topological defects such as walls, strings, monopoles, or textures may have been produced through the so-called Kibble mechanism [1]. Among them cosmic strings or global textures may help large-scale structure formation if their energy scale is $O\left(10^{16} \mathrm{GeV}\right)[2,3]$. Detection of magnetic monopoles, on the other hand, is an important clue to verify unified theories because they are inevitably produced when the electromagnetic $U(1)$ gauge group branches off at some symmetry breaking $[1,2,4]$.

In discussing these phase transitions, it has been implicitly assumed that the universe was in thermal equilibrium state at least by the GUT era. However, in order to attain thermal equilibrium from an arbitrary initial state of the universe, it is necessary that particle interaction rates exceed the cosmic expansion rate, which is not always possible in such an early stage of cosmic evolution [5]. Hence formation of topological defects with typical GUT scale may not be described by the Kibble mechanism correctly unless the universe started its evolution in a thermal equilibrium state.

It is more natural to expect that our universe started its classical evolution out of a chaotic state governed by quantum and thermal fluctuations [6] and that it underwent inflation to be globally homogeneous and isotropic as observed today [7]. Then it turns out to be after the reheating epoch that the universe was first filled with radiation in thermal equilibrium. Unfortunately, the 
maximum temperature it experienced, or the reheat temperature, may not be so high as the GUT scale generally in order to avoid too much gravitational waves or density fluctuations [8] and/or too many gravitinos [9] to be produced after inflation. On the other hand, models with high enough reheat temperature or extended inflation scenario may not solve the monopole problem, even if they may keep large enough density of strings or textures. Thus it is very difficult to obtain a sensible scenario of the early universe which provides an appropriate initial condition of galaxy formation through topological defects in the grand unification scale.

In order to resolve this difficulty several mechanisms of non-thermal phase transitions have been proposed in which the Higgs field is coupled either with spacetime curvature $\mathcal{R}[10,11]$ or the inflation-driving field $\phi[12,13,14]$. The former mechanism is especially plausible since the effective potential naturally has a finite-curvature correction in the inflating spacetime just as it would have a finite-temperature correction in the hot big bang universe [15]. In these scenarios phase transition takes place during the inflationary stage due to time variation of $\mathcal{R}$ or $\phi$ and it is triggered by quantum fluctuations rather than thermal fluctuations.

While the properties of thermal phase transitions have been extensively studied, those of the above non-thermal phase transitions have not been fully investigated. The purpose of the present paper is to clarify the dynamics of a second-order phase transition during inflation as well as the spectrum of the defects produced. Complementary to the present paper, the case topological defects are produced through a first-order phase transition has been studied by Copeland, Kolb, and Liddle [16] in the context of extended inflation scenario and quantum creation of defects through tunneling during inflation has been investigated by Basu, Guth, and Vilenkin [17].

The rest of the paper is organized as follows. After introducing our model in $\S 2$, we examine when evolution of the scalar field becomes deterministic in §3. Then we estimate the power spectrum of quantum fluctuations in $\S 4$. In $\$ 5$ the result of numerical simulations on the spectrum of defects thus produced is reported and its analytic interpretation is given. Finally $\S 6$ is devoted to discussion and conclusions. 


\section{The model}

In the present paper, for definiteness, we consider the chaotic inflation scenario realized by a massive scalar field $\phi$ with mass $m$ in the spatially flat Friedmann-Robertson-Walker spacetime $d s^{2}=d t^{2}-a(t)^{2} d \mathrm{x}^{2}=a(\eta)^{2}\left(d \eta^{2}-d \mathrm{x}^{2}\right)$. In the inflationary stage, the classical evolution of $\phi(t)$, the scale factor $a(t)$, and the Hubble parameter $H(t)$, is given by

$$
\begin{aligned}
\phi(t) & =\phi_{0}-\frac{m M_{p l}}{2 \sqrt{3 \pi}}\left(t-t_{0}\right), \\
H(t) & =\sqrt{\frac{4 \pi m^{2}}{3 M_{p l}^{2}}} \phi(t)=H_{0}-\frac{m^{2}}{3}\left(t-t_{0}\right), \\
a(t) & =a_{0} \exp \left[\frac{2 \pi}{M_{p l}^{2}}\left(\phi_{0}^{2}-\phi^{2}(t)\right)\right]=a_{0} \exp \left[\frac{3}{2 m^{2}}\left(H_{0}^{2}-H^{2}(t)\right)\right],
\end{aligned}
$$

respectively, where $M_{p l}$ is the Planck mass and $t_{0}$ is arbitrary epoch when $a=a_{0}, \phi=\phi_{0}$ and $H=H_{0}$. The above expression is a good approximation when $\phi$ satisfies the following inequality.

$$
\phi_{e} \equiv \frac{M_{p l}}{\sqrt{4 \pi}} \lesssim \phi(t) \lesssim\left(\frac{3}{32 \pi}\right)^{\frac{1}{4}}\left(\frac{M_{p l}}{m}\right)^{\frac{1}{4}} M_{p l} \equiv \phi_{i}
$$

For $\phi>\phi_{i}$ the evolution of $\phi$ is dominated by quantum fluctuations [18] and at $\phi=\phi_{e}$ the time variation rate of $\phi$ or $\dot{\phi} / \phi$ becomes as large as the expansion rate $H$ so that inflationary expansion terminates. The present horizon scale left the Hubble radius when $\phi \approx 3 M_{p l}$ corresponding to the $e$-folding number of inflation after this epoch to be about $n \approx 60$. In order for the density fluctuations to be small enough on this scale $m$ should satisfy the constraint $m \lesssim 10^{13} \mathrm{GeV}$ [19].

As is seen in (2), for $H(t) \gtrsim m / \sqrt{3}$ or $\phi \gtrsim M_{p l} / \sqrt{4 \pi}$ time variation rate of the Hubble parameter is so small that the evolution of the scale factor is indistinguishable from that in de Sitter spacetime during each span of several expansion times. Hence we may utilize various results of quantum field theory in de Sitter spacetime.

Here we consider the evolution of a scalar field $\chi$ with the Lagrangian

$$
\mathcal{L}_{\chi}=\frac{1}{2}(\partial \chi)^{2}-V[\chi], \quad V[\chi]=\frac{\lambda}{4}\left(\chi^{2}-v^{2}\right)^{2}+\frac{1}{2} \xi \mathcal{R} \chi^{2}
$$

with a positive coupling parameter $\xi$ to the scalar curvature $\mathcal{R}$. Depending on the number of components of $\chi, j$, this system allows a domain wall $(j=1)$, a string $(j=2)$, a monopole 
$(j=3)$, or a texture $(j=4)$ solution if $\xi \mathcal{R} \ll \lambda v^{2}$. In discussing the phase transition, however, we concentrate on the single-component case. Generalization to multi-components cases will be discussed in $\S 6$.

In the inflationary stage the scalar curvature $\mathcal{R}$ is given by

$$
\mathcal{R}=12 H^{2}+6 \dot{H} \cong \frac{16 \pi}{M_{p l}^{2}} m^{2} \phi^{2}-2 m^{2} .
$$

The symmetry of $\chi$ is restored if $\xi \mathcal{R}>\lambda v^{2}$ and second-order phase transition takes place as $\mathcal{R}$ decreases gradually.

\section{Evolution of the scalar fields}

In this section, we trace the evolution of $\chi$ to discuss when its sign in each domain becomes fixed so that we may predict where topological defects will appear after completion of the phase transition. The symmetric state $\chi=0$ becomes classically unstable when $\xi \mathcal{R}$ becomes smaller than $\lambda v^{2}$. Evolution of the scalar field after this epoch can be divided to two stages. In the first stage when its potential at the origin is still nearly flat, quantum fluctuations govern its evolution and its amplitude grows gradually. In the second stage typical amplitude of $\chi$ becomes so large that its dynamics may be determined classically and its fate in each domain becomes predictable. We may regard that $\chi$ 's sign is fixed in most domains at this epoch and follow its evolution classically thereafter.

In terms of the mean square value of a scalar field $\Phi$ with a constant mass $M$ [20],

$$
\left\langle\Phi^{2}(t)\right\rangle \cong \begin{cases}\frac{3 H^{4}}{8 \pi^{2} M^{2}}\left[1-\exp \left(-\frac{2 M^{2} t}{3 H}\right)\right], & \text { for } M^{2}>0 \\ \frac{H^{3}}{4 \pi^{2}} t, & \text { for } M^{2}=0 \\ \frac{3 H^{4}}{8 \pi^{2}\left|M^{2}\right|}\left[\exp \left(\frac{2\left|M^{2}\right| t}{3 H}\right)-1\right], & \text { for } M^{2}<0\end{cases}
$$

it has been naively concluded in the previous literatures $[13,21]$ that the first stage lasts while

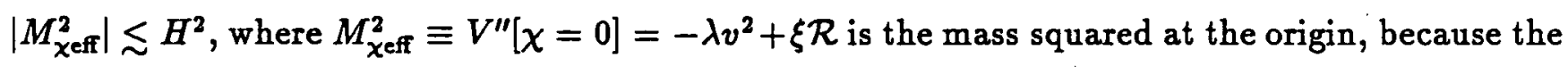
linear growth of mean square field with time is characteristic of the era dominated by long-wave quantum fluctuations. However, this criterion is not valid in the present case in which $M_{\chi \text { eff }}^{2}$ is time-dependent. 
In order to find a more appropriate way to estimate when the evolution of $\chi$ becomes deterministic, let us focus on its long-wavelength part which is responsible for the symmetry breaking and satisfies the Langevin equation

$$
\frac{d \chi}{d u}=-\frac{V^{\prime}[\chi]}{3 H^{2}(u)}+\frac{f(u)}{H(u)}
$$

under the assumption of slow rolling, which is justified if $\left|M_{\text {xeff }}^{2}\right| \ll 9 H^{2}$ [23]. Here we have used a new time variable $u \equiv \ln a(t)-\ln a\left(t_{0}\right)$ which is the $e$-folding number of cosmic expansion from $t_{0}$ to $t$. The Hubble parameter squared is given by $H^{2}(u)=H^{2}(0)-\frac{2}{3} m^{2} u \equiv H_{0}^{2}-\frac{2}{3} m^{2} u$. In the right-hand-side (RHS) of (8), the first term represents classical potential force, while the second term stands for random quantum noise with the correlation $\left\langle f\left(u_{1}\right) f\left(u_{2}\right)\right\rangle \cong \frac{H^{4}\left(u_{1}\right)}{4 \pi^{2}} \delta\left(u_{1}-u_{2}\right)$. The above Langevin equation yields the following equation of motion of $\left\langle\chi^{2}(u)\right\rangle$

$$
\frac{d}{d u}\left\langle\chi^{2}(u)\right\rangle \cong \frac{2 M_{\chi \text { eff }}^{2}}{3 H^{2}(u)}\left\langle\chi^{2}(u)\right\rangle+\frac{H^{2}(u)}{4 \pi^{2}},
$$

where the term proportional to $\left\langle\chi^{4}(u)\right\rangle$ has been neglected, which is shown to be a good approximation at the end of this section. Again the first term of the RHS is classical potential force and the second term represents quantum diffusion without which (9) would be the same as the classical equation of motion of $\chi^{2}$. Thus by comparing magnitudes of the two terms, one may conclude if the evolution of $\chi$ is dominated by potential force or fluctuations in typical domains.

Equation (9) may readily be solved:

$$
\left\langle\chi^{2}(u)\right\rangle=\left[\left\langle\chi^{2}(0)\right\rangle+\frac{H_{0}^{2}}{4 \pi^{2}} \int_{0}^{u} e^{8 \xi u^{\prime}}\left(1-\frac{2 m^{2} u^{\prime}}{3 H_{0}^{2}}\right)^{\frac{\lambda \nu^{2}}{m^{2}}+2 \xi+1} d u^{\prime}\right] e^{-8 \xi u}\left(1-\frac{2 m^{2} u}{3 H_{0}^{2}}\right)^{-\frac{\lambda v^{2}}{m^{2}}+2 \xi} .
$$

If $2 m^{2} u \ll 3 H_{0}^{2}$ and $m^{2} \ll \lambda v^{2}$,

$$
\left(1-\frac{2 m^{2} u}{3 H_{0}^{2}}\right)^{\frac{\lambda v^{2}}{m^{2}}+2 \xi+1} \approx \exp \left(-\frac{2 \lambda v^{2}}{3 H_{0}^{2}} u\right)
$$

and (10) reads

$$
\begin{aligned}
\left\langle\chi^{2}(u)\right\rangle \approx\langle & \left\langle\chi^{2}(0)\right\rangle \exp \left[-\frac{2\left(12 \xi H_{0}^{2}-\lambda v^{2}\right)}{3 H_{0}^{2}} u\right] \\
& +\frac{1}{4 \pi^{2}} \frac{3 H_{0}^{4}}{2\left(12 \xi H_{0}^{2}-\lambda v^{2}\right)}\left\{1-\exp \left[-\frac{2\left(12 \xi H_{0}^{2}-\lambda v^{2}\right)}{3 H_{0}^{2}} u\right]\right\} .
\end{aligned}
$$


Since $12 \xi H_{0}^{2}-\lambda v^{2}$ is equal to the mass squared of $\chi, M_{\chi \text { eff }}^{2}[u=0]$, the above result is equivalent to that with a constant mass $M_{\text {xeff }}[u=0]$ calculated by the one-loop field theoretic method in de Sitter spacetime (7) ${ }^{1}$. With the above approximation $(11),\left\langle\chi^{2}(u)\right\rangle$ may increase or decrease depending on the sign of $12 \xi H_{0}^{2}-\lambda v^{2}$ or the choice of $t_{0}$.

We should hence consider higher order terms in order to discuss transition to the second stage. Let us first consider the last exponent of (10),

$$
e^{-8 \xi u}\left(1-\frac{2 m^{2} u}{3 H_{0}^{2}}\right)^{-\frac{\lambda v^{2}}{m^{2}}+2 \xi} \equiv G(u) .
$$

We can expand $\ln G(u)$ as

$$
\begin{aligned}
\ln G(u)= & -8 \xi u+\frac{\lambda v^{2}+2 \xi m^{2}}{m^{2}}\left[\frac{2 m^{2}}{3 H_{0}^{2}} u+\frac{1}{2}\left(\frac{2 m^{2}}{3 H_{0}^{2}} u\right)^{2}+\frac{1}{3}\left(\frac{2 m^{2}}{3 H_{0}^{2}} u\right)^{3}+\ldots\right] \\
= & \frac{2\left(\lambda v^{2}+2 \xi m^{2}-12 \xi H_{0}^{2}\right)}{3 H_{0}^{2}} u+\frac{2 m^{2}\left(\lambda v^{2}+2 \xi m^{2}\right)}{9 H_{0}^{4}} u^{2} \\
& +\frac{\lambda v^{2}+2 \xi m^{2}}{3 m^{2}} O\left(\left(\frac{2 m^{2}}{3 H_{0}^{2}} u\right)^{3}\right) .
\end{aligned}
$$

The approximation (11) corresponds to adopting only the first term of (13). However, in the case $M_{\text {Xeff }}^{2}[u=0]=12 \xi H_{0}^{2}-2 \xi m^{2}-\lambda v^{2}$ is small, in which we are interested, the second term may dominate the first and $G(u)$ grows. For simplicity, let us choose $t_{0}$ so that $M_{\text {zeff }}^{2}=0$ or

$$
H_{0}^{2}=\frac{\left(\lambda v^{2}+2 \xi m^{2}\right)}{12 \xi}
$$

at that time. Then for small $u, G(u)$ is given by

$$
G(u) \cong \exp \left[\frac{32 \xi^{2} m^{2}}{\lambda v^{2}+2 \xi m^{2}} u^{2}\right] .
$$

Thus $G(u)$ starts to grow exponentially at $u \cong u_{f}$ with

$$
u_{f} \equiv \sqrt{\frac{\lambda v^{2}}{32 \xi^{2} m^{2}}}=\sqrt{\frac{c}{32 \xi^{2}}}, \quad c \equiv \frac{\lambda v^{2}}{m^{2}} .
$$

Similarly the integrand of $(10)$, which is denoted $F\left(u^{\prime}\right)$, may be approximated as

$$
\ln F\left(u^{\prime}\right)=-\frac{2 m^{2}\left(m^{2}+2 \xi m^{2}+\lambda v^{2}\right)}{9 H_{0}^{4}}\left[u+\frac{3 H_{0}^{2}\left(\lambda v^{2}+m^{2}+2 \xi m^{2}-12 \xi H_{0}^{2}\right)}{2\left(m^{2}+2 \xi m^{2}+\lambda v^{2}\right) m^{2}}\right]^{2}
$$

\footnotetext{
${ }^{1}$ The equivalence between stochastic inflation method and field theoretic method has been discussed in [24].
} 


$$
\begin{aligned}
& +\frac{\left(\lambda v^{2}+m^{2}+2 \xi m^{2}-12 \xi H_{0}^{2}\right)^{2}}{2\left(m^{2}+2 \xi m^{2}+\lambda v^{2}\right) m^{2}}-\frac{\lambda v^{2}+m^{2}+2 \xi m^{2}}{3 m^{2}} O\left(\left(\frac{2 m^{2}}{3 H_{0}^{2}} u\right)^{3}\right) \\
\cong & -\frac{32 \xi^{2} m^{2}}{\lambda v^{2}}\left(u+\frac{1}{8 \xi}\right)^{2}+\frac{m^{2}}{2 \lambda v^{2}},
\end{aligned}
$$

where in the last expression we have used our assumption of $\lambda v^{2} \gg m^{2}$. Thus the integral yields

$$
\int_{0}^{u} F\left(u^{\prime}\right) d u^{\prime} \approx \begin{cases}u & u \lesssim u_{f}, \\ u_{f} & u \gtrsim u_{f},\end{cases}
$$

The next task is to estimate $\left\langle\chi^{2}(0)\right\rangle$, which may depend on the initial state of the universe in general. One may expect, however, that the amplitude of $\left\langle\chi^{2}\right\rangle$ is $O\left((H / 2 \pi)^{2}\right)$ at the epoch when $M_{\chi \text { eff }}^{2}=H^{2}$, independent of the initial condition, if the inflationary expansion lasts long enough by that time. We have numerically calculated $\left\langle\chi^{2}(0)\right\rangle$ using (10) starting with this condition for various values of $c=\lambda v^{2} / m^{2}$ ranging from 5 to 200 and $\xi=3 / 16$. (The reason why we adopted $3 / 16$ as $\xi$ will become evident in the next section). As a result the inequality

$$
\left\langle\chi^{2}(0)\right\rangle \lesssim \frac{H_{0}^{2}}{4 \pi^{2}} \sqrt{\frac{\lambda v^{2}}{m^{2}}}
$$

was always satisfied. Hence $\left\langle\chi^{2}(0)\right\rangle$ is unimportant in (10) for $u \gtrsim u_{f}$ and we may conclude

$$
\left\langle\chi^{2}(u)\right\rangle \approx \frac{H_{0}^{2}}{4 \pi^{2}} \sqrt{\frac{\lambda v^{2}}{32 \xi^{2} m^{2}}} \exp \left(\frac{32 \xi^{2} m^{2}}{\lambda v^{2}} u^{2}\right) .
$$

Inserting this into (9), we find that classical potential force begins to dominate quantum fluctuation at $u \approx u_{f}$

The condition $u=u_{f}$ is realized at

$$
t=t_{0}+\frac{3}{m}\left(\frac{c+2 \xi}{12 \xi}-\sqrt{\frac{c}{12 \xi}+\frac{1}{6}-\frac{1}{6 \xi} \sqrt{\frac{c}{2}}}\right) \equiv t_{f}
$$

when the Hubble parameter,

$$
H=m \sqrt{\frac{c}{12 \xi}+\frac{1}{6}-\frac{1}{6 \xi} \sqrt{\frac{c}{2}}} \equiv H_{f}
$$

is related with $M_{\text {xeff }}^{2}$ as

$$
\frac{H_{f}^{2}}{\left|M_{\chi \mathrm{eff}}^{2}\right|}=\frac{1}{12 \sqrt{2} \xi}\left(\sqrt{c}-\sqrt{2}+\frac{2}{\sqrt{c}} \xi\right)
$$


One can estimate the $e$-folding number of inflation after $t=t_{f}$ as

$$
n_{f e}=\frac{3}{2 m^{2}}\left(H_{f}^{2}-\frac{m^{2}}{3}\right)=\frac{c}{8 \xi}-\frac{1}{4}-\frac{1}{4 \xi} \sqrt{\frac{c}{2}} .
$$

In order that topological defects, which are thus produced, may leave observable traces, $n_{f e}$ should be smaller than $\approx 60$, otherwise they are inflated away from the present horizon. Hence this sets an upper bound on $c$. On the other hand, it should be at least larger than zero for the above arguments to be valid. Thus $c$ should satisfy the following inequality for $\xi=3 / 16$.

$$
3 \lesssim c \lesssim 90
$$

So far we have entirely neglected contribution of $\left\langle\chi^{2}(u)\right\rangle$ to $M_{\chi \text { eff }}^{2}$ and the term proportional to $\left\langle\chi^{4}(u)\right\rangle=O\left(\left\langle\chi^{2}(u)\right\rangle^{2}\right)$ in (9). It is justified if

$$
\lambda\left\langle\chi^{2}(u)\right\rangle \approx \frac{\lambda^{2} v^{2}}{128 \sqrt{2} \pi^{2} \xi^{3}} \sqrt{\frac{\lambda v^{2}}{m^{2}}} \lesssim m^{2},
$$

which we may regard as a constraint on $\lambda$ :

$$
\lambda \lesssim 128 \sqrt{2} \pi^{2} \xi^{3} c^{-\frac{3}{2}} .
$$

It is easy to satisfy the above inequality. Even if we take a rather large value of $c$, say $c=90$, it only requires that $\lambda \lesssim 10^{-2}$ when $\xi=3 / 16$.

\section{Power spectrum of fluctuations}

Had there been no correlation beyond the Hubble horizon, we could have identified the correlation length of the phase transition with the Hubble length at $t=t_{f}$. However, the scalar field is correlated on various scales due to the inflationary expansion, which is characterized by the power spectrum $\left|\chi_{k}\right|^{2}$ of long-wave quantum fluctuations given in terms of mode function $\chi_{k}(t)$. Here we calculate it.

We decompose the operator $\chi$ as

$$
\chi(\mathbf{x}, t)=\int \frac{d^{3} k}{(2 \pi)^{3}}\left[a_{\mathbf{k}} \chi_{k}(t) e^{i \mathbf{k x}}+a_{\mathbf{k}}^{\dagger} \chi^{*}(t) e^{-i \mathbf{k x}}\right],
$$


where $a_{k}$ and $a_{k}^{\dagger}$ are annihilation and creation operators, respectively, and the mode function satisfies the following equation of motion.

$$
\ddot{\chi}_{k}(t)+3 H \dot{\chi_{k}}(t)+\left(\frac{k}{a(t)}\right)^{2} \chi_{k}(t)+M_{\chi_{\text {eff }}}^{2} \chi_{k}(t)=0 .
$$

Using a new variable $\bar{\chi}_{k} \equiv a^{\frac{3}{2}}(t) \chi_{k}$ the above equation is rewritten as

$$
\left[\frac{\partial^{2}}{\partial t^{2}}+\left(\frac{k}{a(t)}\right)^{2}+M_{\chi \text { eff }}^{2}-\frac{3}{2} \dot{H}-\frac{9}{4} H^{2}\right] \bar{\chi}_{k}=0
$$

In the inflationary stage, when slow rolling condition $|\dot{H}| \ll H^{2}$ is applicable, we may regard the Hubble parameter to be constant in several expansion time scales during which physical wave number $k / a(t)$ decreases exponentially. Moreover since we are interested in the regime $\left|M_{\text {xeff }}^{2}\right| \lesssim H^{2}$ where quantum fluctuations are important, equation (27) can be approximately solved by the Hankel function of rank $3 / 2$ and the positive frequency mode corresponding to that in the Minkowski vacium for $k \gg H a(t)$ reads

$$
\tilde{\chi}_{k}(t) \cong \sqrt{\frac{\pi}{4 H}} H_{\frac{3}{2}}^{(1)}\left(\frac{k}{H a(t)}\right)
$$

where the mode function $\tilde{\chi}_{k}(t)$ is appropriately normalized according to

$$
\tilde{\chi}_{k}(t) \dot{\bar{\chi}}_{k}^{*}(t)-\tilde{\chi}_{k}^{*}(t) \dot{\bar{\chi}}_{k}(t)=i
$$

While the above expression (28) is a good approximation, the comoving mode $k$ can shift from short-wavelength regime to long-wavelength regime. In the latter phase, in which $(k / a(t))^{2}$ is negligible in (27), one may solve it by means of the WKB method [22].

$$
\tilde{\chi}_{k}(t) \cong A_{k} a^{\frac{3}{2}}\left(t_{k}\right)\left(\frac{S\left(t_{k}\right)}{S(t)}\right)^{\frac{1}{2}} \exp \left[\int_{t_{k}}^{t} S\left(t^{\prime}\right) d t^{\prime}\right]+B_{k} a^{\frac{3}{2}}\left(t_{k}\right)\left(\frac{S\left(t_{k}\right)}{S(t)}\right)^{\frac{1}{2}} \exp \left[-\int_{t_{k}}^{t} S\left(t^{\prime}\right) d t^{\prime}\right]
$$

where

$$
S(t) \equiv \frac{3}{2} H\left(1-\frac{4 M_{\text {xeff }}^{2}}{9 H^{2}}+\frac{2 \dot{H}}{3 H^{2}}\right)^{\frac{1}{2}} \cong \frac{3}{2} H .
$$

The WKB approximation is valid provided $|\dot{S}| \ll S^{2}$. In the present model $S(t)$ is explicitly written as

$$
\dot{S(t)}=\frac{3}{2} H(t)\left(1-\frac{16}{3} \xi+\frac{(8 \xi-2) m^{2}+4 \lambda v^{2}}{9 H^{2}(t)}\right)^{\frac{1}{2}}
$$


In the case $\xi=3 / 16=0.1875$, which is slightly larger than the conformally coupled case $\xi=$ $1 / 6=0.166 \ldots, S(t)$ is time-independent:

$$
S=\sqrt{\lambda v^{2}-\frac{3}{8} m^{2}}
$$

This is why we have taken $\xi=3 / 16$ in $\S 3$ and, for simplicity, we will mainly study this case in the following as well.

The coefficients $A_{k}$ and $B_{k}$ may be determined by the initial condition at $t=t_{k}$ and normalization condition. One may determine the coefficient of the leading term $A_{k}$ with the help of (28), which is explicitly written as

$$
\begin{aligned}
\bar{\chi}_{k}(t) & \cong-i\left(\sqrt{\frac{H^{2}}{2 k^{3}}} a^{\frac{3}{2}}(t)-\frac{i}{\sqrt{2 k}} a^{\frac{1}{2}}(t)\right) e^{-i \frac{k}{H a(t)}} \\
& \approx-i \sqrt{\frac{H^{2}}{2 k^{3}}} a^{\frac{3}{2}}(t)
\end{aligned}
$$

where the last expression is the approximate solution for the case the physical wavelength of $k$ mode is larger than the horizon scale $(k \lesssim a H)$. With the approximation $S(t) \cong \frac{3}{2} H(t),(30)$ reads

$$
\bar{\chi}_{k}(t) \cong A_{k} a^{\frac{3}{2}}\left(t_{k}\right) \exp \left[\frac{3}{2} H\left(t-t_{k}\right)\right] \cong A_{k} a^{\frac{3}{2}}(t) .
$$

Thus from (34) and (35) we can determine $A_{k}$ as

$$
A_{k}=\sqrt{\frac{H^{2}\left(t_{k}\right)}{2 k^{3}}}
$$

where the unimportant phase factor has been omitted. Thus the mode function in the longwavelength regime is given as

$$
\chi_{k}(t) \cong \sqrt{\frac{H^{2}\left(t_{k}\right)}{2 k^{3}}}\left(\frac{S\left(t_{k}\right)}{S(t)}\right)^{\frac{1}{2}}\left(\frac{a\left(t_{k}\right)}{a(t)}\right)^{\frac{3}{2}} \exp \left[\int_{t_{k}}^{t} S\left(t^{\prime}\right) d t^{\prime}\right] .
$$

One may get this result independently of the choice of the connecting time $t_{k}$ as far as both (28) and (30) are reasonable approximations at $t=t_{k}$. Hereafter we take $t_{k}$ to be the epoch $k$-mode leaves the de Sitter horizon, that is,

$$
k=H\left(t_{k}\right) a\left(t_{k}\right) \equiv H_{k} a_{k} .
$$


For the case $\xi=3 / 16,(37)$ reads

$$
\chi_{k}(t)=\sqrt{\frac{H_{k}^{2}}{2 k^{3}}} \exp \left\{-\frac{9}{4 m^{2}}\left[H_{k}^{2}-H^{2}(t)\right]+\frac{3}{m^{2}} \sqrt{\lambda v^{2}-\frac{m^{2}}{8}}\left[H_{k}-H(t)\right]\right\} .
$$

This mode function begins growing at

$$
H(t)=\frac{2}{3} \sqrt{\lambda v^{2}-\frac{m^{2}}{8}} \equiv H_{c}
$$

at which epoch $M_{\text {xeff }}^{2}=-\frac{m^{2}}{2}$. Because of the gradual decrease of the Hubble parameter, it is slightly after $\chi=0$ becomes unstable that $\chi_{k}$ starts to grow.

The amplitude of (39) in the case $\xi=3 / 16$ and $c=5$ is shown in Fig. 1, which depicts relative amplitude to that of a massless minimally-coupled scalar field in the exact de Sitter spacetime given by $\left(H_{f}^{2} / 2 k^{3}\right)^{\frac{1}{2}}$.

\section{Distribution of the topological defects}

\subsection{Method and results of numerical simulations}

Having estimated when evolution of $\chi$ becomes dominated by the potential force in $\S 3$ and calculated the power spectrum of its fluctuations at an arbitrary time in $\S 4$, we are now in a position to apply them to find the spectrum of topological defects produced. Following our two step approximation described in $\S 3$, this is accomplished by calculating spatial distribution of the scalar field at $t=t_{f}$. Thanks to the properties of the vacuum state realized as a result of inflationary expansion, $\chi\left(\mathbf{x}, t_{f}\right)$ is classically given by summing up its Fourier modes as

$$
\chi\left(\mathbf{x}, t_{f}\right)=\sum_{\mathbf{k}} \delta \chi_{\mathbf{k}}\left(\mathbf{x}, t_{f}\right)+\text { C.C. } ; \quad \delta \chi_{\mathbf{k}}\left(\mathbf{x}, t_{f}\right)=\left|\delta \chi_{k}\left(t_{f}\right)\right| e^{i \mathbf{k x}+i \varphi_{\mathbf{k}}},
$$

where $\left|\delta \chi_{\mathbf{k}}\left(t_{f}\right)\right|$ is a random value which has a Gaussian distribution with dispersion $\left|\chi_{k}\left(t_{f}\right)\right|^{2}$ and $\varphi_{\mathbf{k}}$ is a random phase-factor.

If we find the sign of $\chi$ positive (negative) at a certain point at $t=t_{f}$, it will fall down to the minimum of $\chi=+v(-v)$ as phase transition proceeds, since its dynamics has been deterministic by this time in typical domains. Thus we may consider that domain walls are produced between two regions with opposite signs of $\chi$ at this time. 
We have first done three-dimensional simulations as illustrated in figure $2 \mathrm{~A}$, in which we have calculated the value of $\chi\left(x, t_{f}\right)$ through (41) at each point of $32^{3}$ lattices using the power spectrum of $\chi_{k}$ with $\xi=3 / 16$ and $c=5$. We adopted fast Fourier-transform (FFT) method to speed up the calculation [25] and summed up $32^{3}$ modes of $\delta \chi_{\mathbf{k}}$ with $k$ ranging from $2 \pi H_{f} / 32$ to $2 \pi H_{f}$, where $H_{f}^{-1}$ is the separation of neighboring lattice-points taken to be the Hubble length at $t=t_{f}$. In the figure dotted points will fall in the plus minimum or $\chi=v$ and empty points in the minus minimum after the phase transition so that domain walls will be present between them. As is seen there, there are structures on various scales. This is in contrast to figure $2 \mathrm{~B}$, in which the sign of $\chi$ has been randomly assigned to each lattice-point corresponding to the case of a thermal phase transition with the correlation length $H_{f}^{-1}$.

In both cases, however, simulation boxes are dominated by walls with infinitely large surface area. This is simply because both states of $\chi= \pm v$ are realized with equal probability and hence it is unlikely that walls are so distributed that islands of plus minimum exist in the sea of minus minimum or vice versa. Thus it is not appropriate to examine the area-distribution of walls in order to discuss the character of phase transitions.

Hence we instead focus on distribution of separation of each wall. For this purpose we do not have to calculate the value of (41) at all points in three dimensional space. But all we should find is its value along a line, which enables us to employ a much larger simulation box. In fact we have used a box with $\left(2^{13}\right)^{3}$ lattice points and examined the distribution of domains along a line with $2^{13}$ points. We have done simulations for the following two cases:

- A. Power spectrum is given by (39) with $\xi=3 / 16$ and $c=5$.

- B. A scale-invariant power spectrum,

$$
\left|\chi_{k}\right|^{2}=\frac{H_{f}^{2}}{2 k^{3}}
$$

is assigned corresponding to the case with a massless scalar field in de Sitter spacetime.

In both cases we have summed $2^{13} \times 100=819200$ independent mode functions in (41) with $k$ ranging from $2 \pi H_{f} / 2^{13}$ to $2 \pi H_{f}$. Simulations have also been done in the case a box with $\left(2^{12}\right)^{3}$ 
points. As a result no artificial boundary effect was observed. In addition simulations in which the upper bound of $k$ is taken to be $\pi H_{f}$ have been carried out and no effect of small-scale cutoff emerged. For comparison we have also studied the case

- C. Sign of $\chi$ is assigned at random at each point.

We have done simulations for 500 times for each case and their results are depicted in Figure 3(A. C). We can obtain various consequences from the graph. As is seen there domain-size distribution in phase transition during inflation ( $A$ and $B$ ) is again very different from that in thermal phase transition (C). In the latter case we can fit the distribution with an exponential function

$$
n\left(s, t_{f}\right) d s \propto \exp (-0.67 s) d s,
$$

where $n\left(s, t_{f}\right)$ is the number density of domains with separation $s \sim s+d s$. This is simply because the number of domains with size $s H_{f}^{-1}$ is proportional to the probability of having the same sign of $\chi$ for $s$ times in succession:

$$
n\left(s, t_{f}\right) d s \propto\left(\frac{1}{2}\right)^{\prime} d s \propto \exp (-\ln 2 \cdot s) d s \propto \exp (-0.67 s) d s .
$$

Thus our numerical simulation agrees with the analytic estimate.

On the other hand, in the case B we may fit the distribution with a power-law

$$
n\left(s, t_{f}\right) d s \propto s^{-p} d s, \quad \text { with } p=3.0 .
$$

We can extend the above one-dimensional result into three dimension as follows. First let us define the effective correlation volume of a domain between two walls with separation $s$ by $V \equiv s^{3}$. Then, since the probability to find a structure with this correlation volume by our simulation is proportional to its effective surface area or $s^{2}$, number density of domains with correlation volume $V \sim V+d V, n\left(V, t_{f}\right)$, is related with $n\left(s, t_{f}\right)$ by

$$
n\left(s, t_{f}\right) d s \propto \frac{n\left(V, t_{f}\right)}{s^{2}} d V .
$$

Thus we have

$$
n\left(V, t_{f}\right) d V \propto n\left(s, t_{f}\right) s^{2} d s \propto n\left(s, t_{f}\right) d^{3} s \propto \frac{d^{3} s}{s^{3}} .
$$


Therefore in the case scale-invariant fluctuation is assigned, we find that the spectrum of the resultant topological defects is also scale-invariant. Analytic arguments to derive this distribution will be given in the next subsection.

Finally the plot A in Fig. 3, for which more realistic spectrum has been assigned, has almost the same profile as the plot $B$ for $s \lesssim 80$ but larger domains are suppressed because amplitudes of fluctuations with larger wavelength are smaller. From Figs. 1 and 3 we may conclude the fluctuation mode with $\sqrt{k^{3}\left|\chi_{k}\left(t_{f}\right)\right|^{2}}$ smaller than $\sim 10 \%$ of its maximum is unimportant and the spectrum of topological defects on such a large scale should approach that predicted by white-noise fluctuations.

\subsection{Analytic interpretation of a scale-invariant spectrum}

Here let us consider how the above scale-invariant feature of topological defects is explained analytically. To make the following discussion simple and clear let us focus on a patch of space which was homogeneous with $\chi=0$ at $t=0$. Let us further assume that the space may be divided into many domains of horizon volume $\mathrm{H}^{-3}$ with different values of $\chi$ at each time. Then probability distribution function of $\chi$ is given by

$$
P[\chi(\mathbf{x}, t)=\chi]=\frac{1}{\sqrt{2 \pi G(0, t)}} \exp \left[-\frac{\chi^{2}}{2 G(0, t)}\right] ; \quad G(0, t)=\left\langle\chi^{2}(\mathbf{x}, t)\right\rangle=\frac{H^{2}}{4 \pi^{2}} H t .
$$

Furthermore joint probability distribution reads

$$
P\left[\chi\left(\mathbf{x}_{i}, t\right)=\chi_{i} ; \chi\left(\mathbf{x}_{j}, t\right)=\chi_{j}\right]=\frac{1}{2 \pi G(0, t) \sqrt{1-\rho^{2}(r, t)}} \exp \left\{-\frac{\chi_{i}^{2}+\chi_{j}^{2}-2 \rho(r, t) \chi_{i} \chi_{j}}{2 G(0, t)\left[1-\rho^{2}(r, t)\right]}\right\},
$$

where $\rho(r, t) \equiv G(r, t) / G(0, t)=\left\langle\chi\left(\mathbf{x}_{i}, t\right) \chi\left(\mathbf{x}_{j}, t\right)\right\rangle / G(0, t)$ with $\left|\mathbf{x}_{i}-\mathbf{x}_{j}\right|=r$. The condition of existence of a wall at a certain point is that $\chi$ changes its sign there, whose probability, $F(t)$, is given by

$$
\begin{aligned}
F(t) & \equiv \int_{-\infty}^{0} d \chi_{i} \int_{0}^{+\infty} d \chi_{j} 2 P\left[\chi\left(\mathbf{x}_{i}, t\right)=\chi_{i} ; \chi\left(\mathbf{x}_{j}, t\right)=\chi_{j}\right] ; \quad\left|\mathbf{x}_{i}-\mathbf{x}_{j}\right|=H^{-1}, \\
& =\frac{1}{\pi} \arccos \left[\frac{G\left(H^{-1}, t\right)}{G(0, t)}\right] \cong \frac{1}{\pi} \arccos \left(1-\frac{1}{H t}\right) \cong \frac{1}{\pi} \sqrt{\frac{2}{H t}}
\end{aligned}
$$

where the last approximation is justified if $H t \gg 1$. Thus $F(t)$ depends on $t$ only weakly even though background space is expanding exponentially. 
By assumption of homogeneity at $t=0$, the largest possible wall at time $t$ has a physical size of $s=H^{-1} e^{H t}$, so that $F(t)$ is related with $n(s, t)$ as

$$
F(t) \approx \int_{H^{-1}}^{H^{-1} e^{H t}} n(s, t) s^{2} d s
$$

where $t$-dependence of $n(s, t)$ reflects the fact that quantum fluctuations are generated continuously to produce and destroy topological defects successively in the course of cosmic expansion. Since this is a rather stationary process without any exponential instability, $n(s, t)$ should not depend on $t$ exponentially just as $F(t)$ does not. Thus from (48) we expect that $n(s, t)$ should be proportional to $s^{-3}$ to eliminate the exponential dependence on $t$ of the upper bound of the integral. In this way we conclude that distribution of walls is scale-invariant and given by

$$
n(s, t) s^{2} d s \approx \frac{1}{(H t)^{\frac{3}{2}}} \frac{d^{3} s}{s^{3}}
$$

for $H t \gg 1$.

\section{Discussion and Conclusions}

We have studied generic features of a second-order phase transition during inflation which is a more natural and attractive scenario to produce topological defects at GUT scale than the Kibble mechanism. We have considered a specific model in which $\chi$ is coupled with spacetime curvature $\mathcal{R}$ and phase transition takes place due to gradual decrease of the Hubble parameter. In this model we have clarified the nature of long-wavelength quantum fluctuations by explicitly calculating the power spectrum and $\left\langle\chi^{2}\right\rangle$.

In terms of the latter quantity we determined when classical potential force surpasses quantum fluctuations in typical domains and investigated the distribution of the field at that epoch through numerical calculations, by which we examined distribution of topological defects.

As a result we have found that they have an almost scale-invariant distribution with smalland large-scale cutoffs. The former is naturally given by the comoving scale corresponding to the Hubble radius at the epoch time evolution of $\chi$ becomes dominated by classical potential force, while our simulations have shown that the latter corresponds to the scale on which the amplitude 
$\sqrt{k^{3}\left|\chi_{k}\left(t_{f}\right)\right|^{2}}$ of fluctuations becomes smaller than $\sim 10 \%$ of its maximum value as is seen in Figs. 1 and 3. On the larger scale the fluctuation amplitude becomes so small that the distribution should approach white-noise spectrum.

Note that though Hodges and Primack reported similar results recently, i.e., scale-invariant distribution of topological defects produced during inflation, their treatment is not satisfactory in two aspects. First they have assumed that topological defects with a certain comoving scale are produced only when that scale leaves the Hubble radius during inflation. Contrary to their claim, however, defects are produced and destroyed on various scales continuously through successive generation of fluctuations as discussed in §5. Second they have not given cutoffs of the distribution explicitly. As is seen above we have significantly improved these points through our analyses.

Finally we stress that our basic results are also applicable to other topological defects such as strings or textures in which we are more interested. For example, strings are loci on which both real and imaginary parts of string-forming complex scalar field changes sign. Hence if self coupling $\lambda$ of the field is small enough we can identify strings with intersections of two different kinds of independent "domain walls." Thus with the same reasoning as $\$ 5.2$ they also have a scale-invariant distribution with cutoffs. Furthermore, since the model parameters must satisfy

$$
\frac{\lambda v^{2}}{m^{2}} \lesssim 90
$$

in order that strings thus produced are not diluted too much by subsequent inflation, we have

$$
\lambda \lesssim 10^{-4}\left(\frac{v}{10^{16} \mathrm{GeV}}\right)^{-2}\left(\frac{m}{10^{13} \mathrm{GeV}}\right)^{2},
$$

so that the condition of $\lambda$ being small is automatically satisfied for cosmologically important strings.

Thus unlike the conventional scenario, strings or textures in the present model have almost scale-invariant spectrum from the beginning without any evolution but can serve as seeds for galaxy formation as well under natural choices of model parameters. 
We thank K. Sato for stimulating discussions and continuous encouragement. JY is grateful to B. Gradwohl and M. Sasaki for valuable discussions and to A. Albrecht for helpful comments. He would also like to thank E. Kolb for hosting his visit to Fermilab and JSPS for a fellowship. Simulations presented here were carried out on the HITAC M-680 at the Computer Center of the University of Tokyo and FACOM M780/10S at the Astronomical Data Analysis Center of National Astronomical Observatory of Japan. This research was supported in part by the Japanese Grant in Aid for Science Research Fund of the Ministry of Education, Science and Culture (No.02740189) and by the NASA (NAGW-1340) and the DOE at Fermilab.

\section{References}

[1] .T.W.B. Kibble, J. Phys. A9(1976)1387

[2] A. Vilenkin, Phys. Rep. 121(1985)265

[3] N. Turok, Phys. Rev. Lett. 63(1989)2625

[4] J.P. Preskill, Ann. Rev. Nucl. Part. Sci. 34(1984)461

[5] J. Ellis and G. Steigman, Phys. Lett. B 89(1980)186

[6] A.D. Linde, Phys. Lett. B 129(1983)177

[7] K. Sato, Mon. Not. R. astr. Soc. 195(1981)467;

A.H. Guth, Phys. Rev. D23(1981)347;

M.S. Turner, presented by J. Audouze and J. Tran Thanh Van, Proc. Cargese School on Fundamental Physics and Cosmology (Editions Frontieres, 1985);

K.A. Olive, Phys. Rep. 190(1990)307

[8] M.D. Pollock, Phys. Lett. B 185(1987)34

[9] M. Kawasaki and K. Sato, Phys. Lett. B 189(1987)23 
[10] J. Yokoyama, Phys. Lett. B 212(1988)273

[11] J. Yokoyama, Phys. Rev. Lett. 63(1989)712

[12] Q. Shafi and A. Vilenkin, Phys. Rev. D29(1984)1870

[13] E.T. Vishniac, K.A. Olive and D. Seckel, Nucl. Phys. B289(1987)717

[14] L.A. Kofman and A.D. Linde, Nucl. Phys. B282(1987)555

[15] G.M. Shore, Ann. of Phys. 128(1980)376;

B. Allen, Nucl. Phys. B226(1983)228

[16] E.J. Copeland; E.W. Kolb, and A.R. Liddle, Phys. Rev. D42(1990)2911

[17] R. Basu, A.H. Guth, and A. Vilenkin, preprint TUTP-91-3 (1991)

[18] A.D. Linde, Mod. Phys. Lett. A1(1986)81

[19] D. Lyth, Phys. Lett. B. 246(1990)359

[20] T.S. Bunch and P.C.W. Davies, Proc. R. Soc. Lond. A 360(1978)117;

A. Vilenkin and L. Ford, Phys. Rev. D26(1982)1231;

A.D. Linde, Phys. Lett. B 116(1982)335

[21] H.M. Hodges and J.R. Primack, Phys. Rev. D43(1991)3155

[22] D.S. Salopek, J.R. Bond and J.M. Bardeen, Phys. Rev. D40(1989)1753

[23] A.A. Starobinsky, Current topics in field theory, quantum gravity and strings, Lecture Notes in Physics 246(Springer, 1986) p.107

[24] M. Sasaki, Y. Nambu, and K. Nakao, Nucl. Phys. B308(1988)868

[25] W.H. Press, B.P. Flannery, S.A. Teukolsky and W.T. Vetterling, Numerical Recipes (Cambridge University Press, 1986) p.390 


\section{Figure Captions}

Fig. 1 Magnitude of the mode function $\left|\chi_{k}\left(t_{f}\right)\right|$ for $\xi=\frac{3}{16}$ and $c=5$ as expressed in equation (39). Plotted values are relative amplitude to $\left(H_{f}^{2} / 2 k^{3}\right)^{\frac{1}{2}}$. Abscissa corresponds to the wavelength $2 \pi a\left(t_{f}\right) / k$ in unit of $H_{f}^{-1}$.

Fig. 2A Distribution of domains in plus vacuum. Dotted points depict regions with $\chi=+v$. Values of the field are determined by giving correlations specified by (39). The box size is $32^{3}$ and lattice separation is equal to $H_{f}^{-1}$.

Fig. 2B Same as Figure 2A but the sign of $\chi$ at each point is given randomly.

Fig. 3 Distribution of the separation of domain walls. This figure shows the sum of 500 times simulations of the length $2^{13}$. Plotted values are integrated one in each logarithmic interval.

$\mathrm{A}(\mathrm{O}): \chi\left(\mathbf{x}, t_{f}\right)$ is calculated using the power spectrum given by (39).

$\mathrm{B}(\mathrm{X}): \chi\left(\mathbf{x}, t_{f}\right)$ is calculated using the scale-invariant power spectrum. Solid line is the power-law fitting whose power index is $\mathbf{- 2 . 0}$ due to integration.

$\mathrm{C}(\Delta)$ : The sign of $\chi\left(\mathrm{x}, t_{f}\right)$ is assigned randomly with equal probability corresponding to the thermal phase transition with correlation length $H_{f}^{-1}$. The plot fits an exponential function depicted by the dashed line. 
Figure 1

relative amplitude

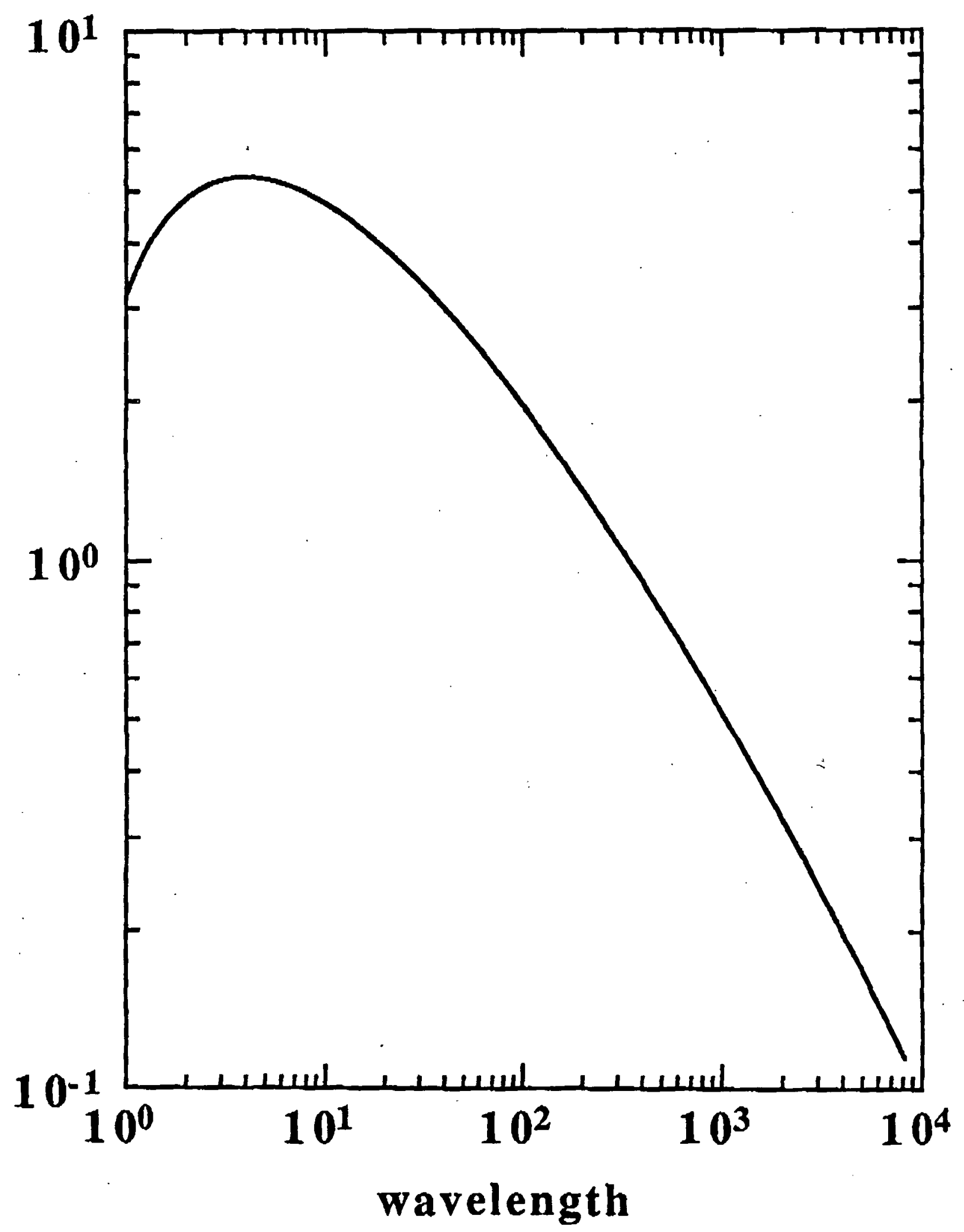




\section{Figure 2A}
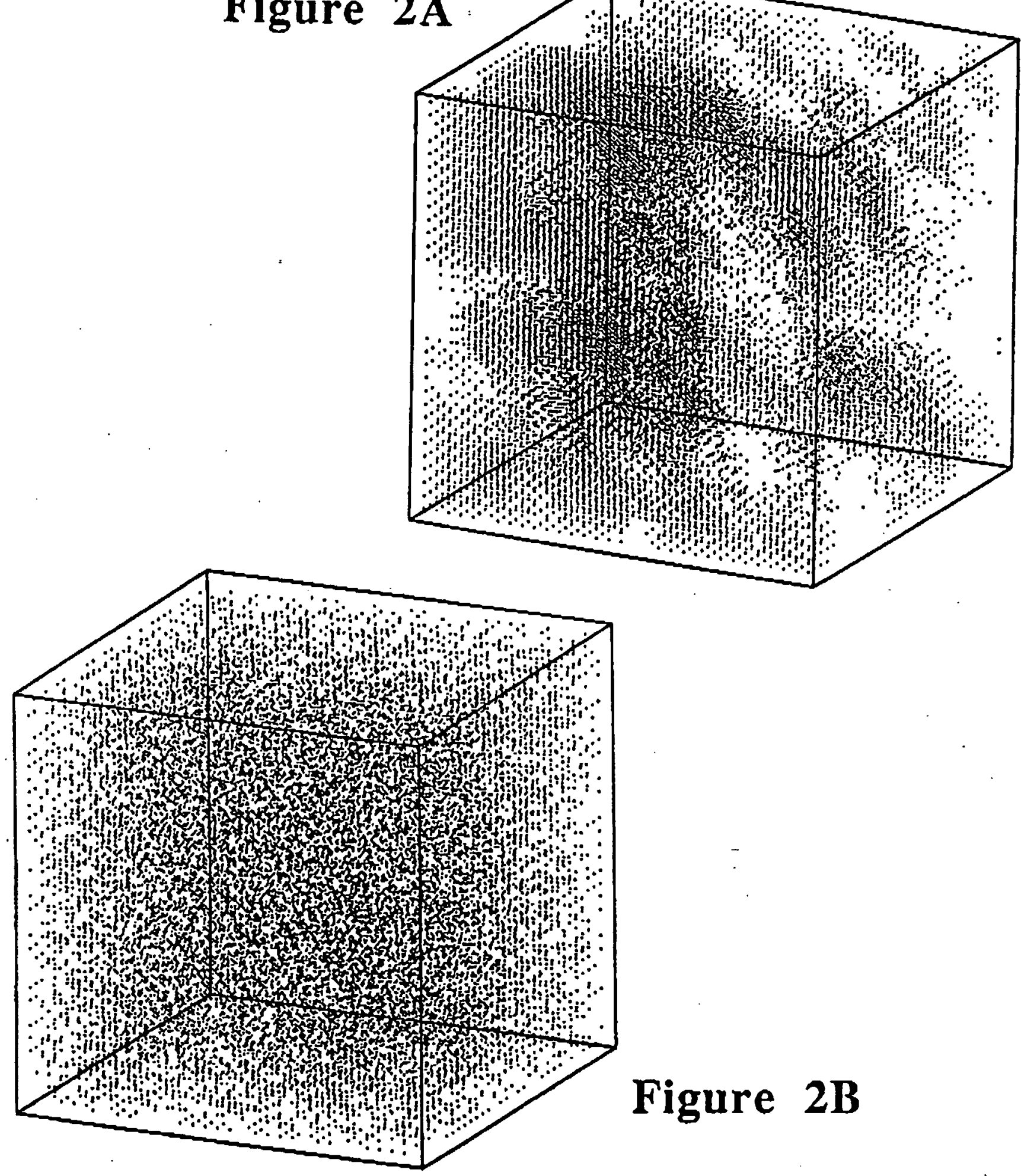

Figure 2B 
Figure 3

number

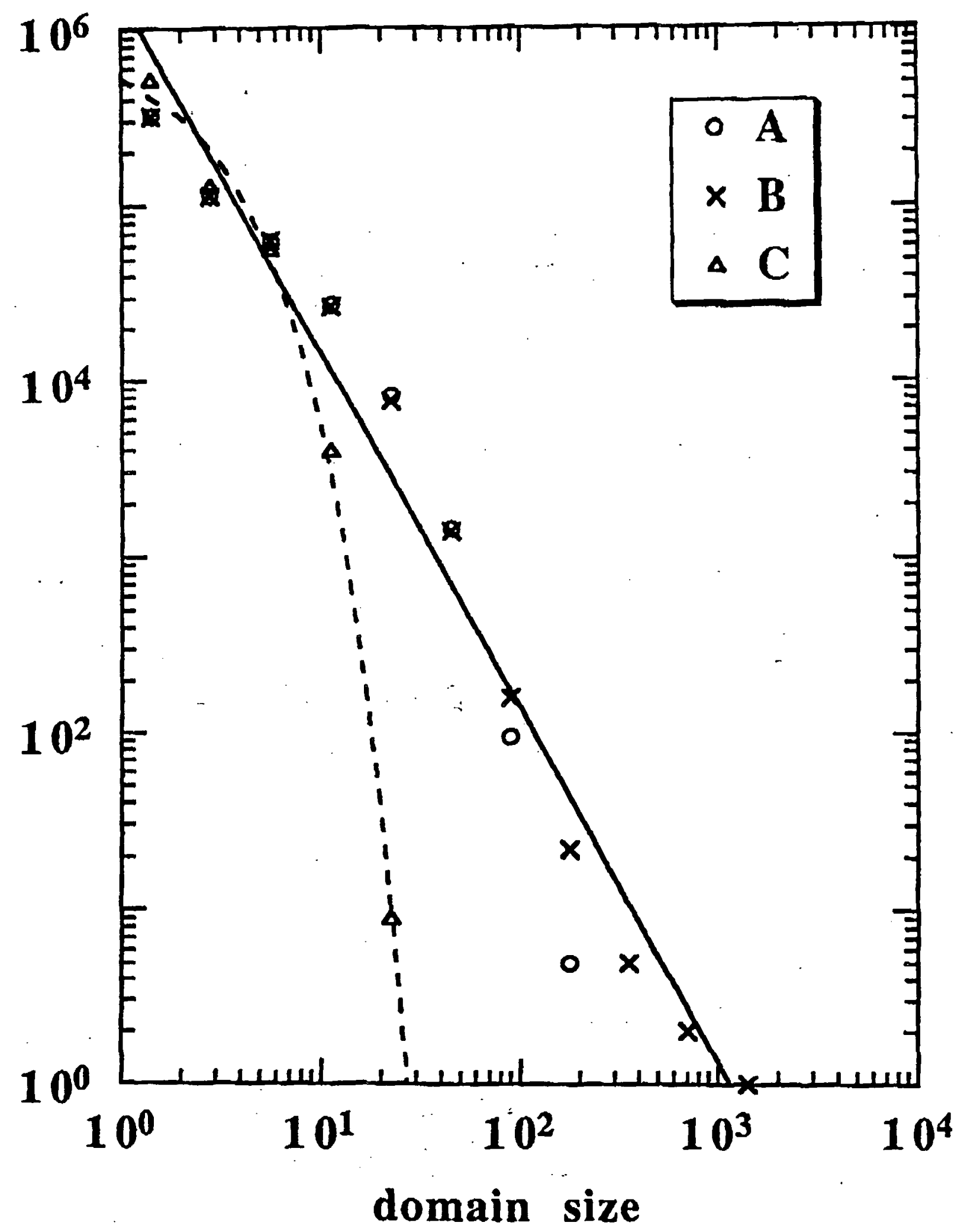

